\title{
Regulated expression of TATA-binding protein-related factor 3 (TRF3) during early embryogenesis
}

\author{
Ye Yang ${ }^{1}$, Jian Cao ${ }^{1}$, Lu Huang ${ }^{1}$, Hai Yan Fang ${ }^{2}$, Hui Zhen Sheng ${ }^{1,2}$ \\ ${ }^{1}$ Laboratory of Stem Cell Biology, Institute of Biochemistry and Cell Biology, Shanghai Institutes for Biological Sciences, Chinese \\ Academy of Sciences, Shanghai 200031, China; ${ }^{2}$ Center for Developmental Biology, Xinhua Hospital, School of Medicine, Shanghai \\ Jiao Tong University, 1665 Kong Jiang Road, Shanghai 200092, China
}

RNA polymerase (Pol) II transcription persists in TATA-box-binding protein $(T B P)^{-/-}$mutant mouse embryos, indicating TBP-independent mechanisms for Pol II transcription in early development. TBP-related factor 3 (TRF3) has been proposed to substitute for TBP in $T B P^{-/-}$mouse embryos. We examined the expression of TRF3 in maturing oocytes and early embryos and found that TRF3 was co-expressed with TBP in the meiotic oocytes and early embryos from the late one-cell stage onward. The amounts of TBP and TRF3 changed dynamically and correlated well with transcriptional activity. Chromatin immunoprecipitation (ChIP) assay revealed that different gene promoters in mouse embryonic stem (ES) cells recruited TRF3 and TBP selectively. Comparative analyses of TRF3 and TBP during cell cycle showed that both factors proceeded through cell cycle in a similar pace, except that TRF3 was slightly delayed than TBP in entering the nucleus when cells were exiting the M-phase. Data from expression and biochemical analyses therefore support the hypothesis that TRF3 plays a role in early mouse development. In addition, results from co-localization study suggest that TRF3 may be also involved in Pol I transcription.

Cell Research (2006) 16:610-621. doi: 10.1038/sj.cr.7310064; published online 23 May 2006

Keywords: TBP, TRF3, oocyte, embryo, ES cell, NIH3T3 cell

\section{Introduction}

TATA-box-binding protein (TBP) is a key component of the general transcription machinery used by all three RNA polymerases [1]. TBP can associate with different sets of TBP-associated factors to form distinct multiprotein complexes; SL1 is required for Pol I, TFIID and B-TFIID for Pol II, and TFIIIB for Pol III [2-4].

To date, three TBP-related factors (TRFs) have been

Correspondence: Hui Zhen Sheng

Tel/Fax: +86-21-55570017;

E-mail: hzsheng2003@yahoo.com

Received 22 Feb 2006; revised 21 Mar 2006; accepted 4 Apr 2006; published online 23 May 2006

Abbreviations: GTF (general transcription factor); TBP (TATA-box-binding protein); TRF (TBP-related factor); ZGA (zygotic gene activation); GV (germinal vesicle); DAPI (4,6-diamidino-2-phenylindole); MEF (mouse embryonic fibroblast); ChIP (chromatin immunoprecipitation) described. TRF1 was identified in Drosophila, and so far is unique to this organism [5]. TRF2, also known as TBP-like factor [6], TBP-like protein [7], and TBP-related protein [8] were found in most metazoans $[9,10]$. TRF2 is essential for the expression of specific zygotic genes at the onset of Pol II transcription in Caenorhabditis elegans, Xenopus laevis, and zebrafish (Danio rerio). In all three organisms, TRF2 depletion resulted in early arrest of embryogenesis and loss of expression of specific genes [11-16]. However, TRF2 is neither expressed [17] nor required for mouse embryogenesis $[18,19]$.

More recently, a fourth member of the TBP family named TRF3 (also called TBP2) has been reported [20-22]. TRF3 has a highly conserved $\mathrm{C}$-terminal domain, which is $96 \%$ conserved with TBP, and a non-conserved $\mathrm{N}$-terminal domain that is $31 \%$ conserved. The TRF 3 gene is present and expressed in most vertebrates, but not lower metazoans. TRF3 binds the TATA box and forms complexes with 
TFIIA and TFIIB. More importantly, TRF3 can mediate transcription initiation in both HeLa cell extract and Xenopus egg extract $[20,22]$. Depletion of TRF3 by antisense RNA injected into fertilized eggs resulted in a change of phenotype in zebrafish, early arrest of embryogenesis in Xenopus laevis, and a change in the expression of specific genes in both species $[20,22]$. These studies show that TRF3 is required for early embryogenesis.

Inactivation of the murine $T B P$ gene by homologous recombination leads to growth arrest and apoptosis at the embryonic blastocyst stage. However, Pol II remains active in the $T B P^{-/-}$cells and the level of Pol II transcription is comparable to those of wild-type cells. In contrast, Pol I and Pol III transcription was arrested [17]. This finding indicates that one or more TBP-independent transcription mechanism(s) exists in early embryos that could play a role in the Pol II transcription. TRF3 has been proposed as a possible candidate $[20,22]$. However, contrary to the studies on TRF3 in amphibian embryos, nothing is known about it in mammalian embryos.

Growing oocytes are transcriptionally active and the level of transcription decreases during oocyte growth [23]. Fully grown oocytes undergo meiotic maturation upon hormonal stimulation. During this process, the oocyte at the germinal vesicle $(\mathrm{GV})$ stage undergoes $\mathrm{GV}$ breakdown, enters the M-phase of the cell cycle, and becomes arrested at metaphase of meiosis II (MII). Transcription is silent during the meiotic division and this transcriptional inertness is maintained until the late one-cell embryo stage in mouse [24]. The decrease in transcriptional activity during oocyte growth is correlated with a decrease in the nuclear concentration of SP1 and TBP [23]. After fertilization, zygotic gene activation (ZGA) occurs in the late one-cell embryo [24-26]. Concomitantly, TBP is detected before the beginning of transcription in the one-cell embryos and the nuclear concentration of TBP increases thereafter [23]. In the mouse, TRF3 is detected in a variety of tissues and cell lines [20]. However, its expression during early development is unknown.

In this study, we compared expression patterns between TRF3 and TBP during early mouse development, in somatic tissues and cells. We found that TRF3 was co-expressed with TBP in both maturing meiotic oocytes and early mouse embryos and that TRF3 proceeded through the mitotic cycle together with TBP, albeit at a slightly different pace. In addition, TRF3 was selectively recruited to promoters of genes in mouse ES cells. These data support the hypothesis that TRF3 has a role in early embryonic development.

\section{Materials and Methods}

Oocyte and embryo collection and culture
White Kunming mice were obtained from the Center for Experimental Animals at the Chinese Academy of Sciences in Shanghai, China. Growing oocytes containing a GV (the GV oocyte) were obtained from 3- to 4-week-old female mice by puncturing ovarian follicles with a 30-G needle in HEPES-buffered Eagle's MEM containing $3 \mathrm{mg} / \mathrm{ml}$ bovine serum albumin (BSA). In all cases, 0.2 $\mathrm{mM}$ 3-isobutyl-1-methyl xanthine was included in the medium to inhibit GV breakdown [27]. The surrounding granulosa cells were separated from the oocytes by pipetting. Fertilized eggs were recovered from ampullae of 6- to 8-week-old female mice that had been superovulated and caged with male mice. Embryos were cultured in CZB medium [28] at $37^{\circ} \mathrm{C}$ in a humidified atmosphere containing $5 \% \mathrm{CO}_{2}$ in air until desired stages (from the early one-cell embryo to blastocyst).

\section{Cell culture}

Murine NIH3T3 embryo fibroblasts were grown in DMEM at $1 \mathrm{~g} / 1$ glucose supplemented with $10 \%$ fetal calf serum, $50 \mathrm{U} / \mathrm{ml}$ penicillin (Sigma), and $50 \mu \mathrm{g} / \mathrm{ml}$ streptomycin. Mouse ES cells (D3 line) were maintained on mitomycin $\mathrm{C}$-inactivated mouse embryonic fibroblast (MEF) feeder layers in medium containing DMEM supplemented with $15 \%$ heat-inactivated fetal bovine serum, $100 \mu \mathrm{M}$ MEM nonessential amino acids, $2 \mathrm{mM}$ glutamine, $0.1 \mathrm{mM}$ 2-mercaptoethanol, $50 \mathrm{U} / \mathrm{ml}$ penicillin, $50 \mu \mathrm{g} / \mathrm{ml}$ streptomycin, and $1000 \mathrm{U} / \mathrm{ml} \mathrm{LIF}$ (ESGRO, Chemicon). Before adding D3 cells, inactivated MEF cells were maintained in MEF growth medium (DMEM supplemented with $2 \mathrm{mM}$ glutamine, $50 \mathrm{U} / \mathrm{ml}$ penicillin, $50 \mu \mathrm{g} / \mathrm{ml}$ streptomycin, and $10 \%$ heat-inactivated fetal bovine serum).

\section{Antibodies}

Rabbit polyclonal antibodies against the mouse TRF3 N-terminal domain (RTRDRQSQLQLPDEHGSELNLNSNSSPDPQSC, residues 100-131) were generated by Abgent (USA). The following antibodies were purchased from Santa Cruz Biotechnology: rabbit anti-human TBP N-terminal domain polyclonal antibody, rabbit antihuman TFIIB polyclonal antibody, rabbit anti-human TFIIA polyclonal antibody, rabbit anti-human TFIIH polyclonal antibody, goat anti-human lamin B1 polyclonal antibody, goat anti-human fibrillarin polyclonal antibody, goat anti-human Pol I polyclonal antibody, and goat anti-human UBF polyclonal antibody. All secondary antibodies were purchased from Jackson Immunoresearch.

\section{Immunofluorescence of oocytes and embryos}

To detect TRF3 in oocytes and early mouse embryos, oocytes and embryos were washed twice in PBS, permeabilized by incubation for $5 \mathrm{~min}$ in $0.1 \%$ Triton X-100 in PBS (PBST) on ice, and fixed for $1 \mathrm{~h}$ in $4 \%$ paraformaldehyde (PFA) in PBS at room temperature. Subsequently, oocytes and embryos were blocked with PBST plus $0.4 \%$ BSA (the blocking solution) for $15 \mathrm{~min}$ and incubated overnight at $4{ }^{\circ} \mathrm{C}$ with the primary antibody diluted in the blocking solution. Oocytes and embryos were washed five times for $15 \mathrm{~min}$ with the blocking solution, incubated with CY3-conjugated anti-rabbit antibody for $1 \mathrm{~h}$ at room temperature, and then washed five times for $15 \mathrm{~min}$ in the blocking solution and once for $5 \mathrm{~min}$ in PBS. Finally, the oocytes and embryos were counterstained with 4,6-diamidino2-phenylindole (DAPI), mounted on glass slides in DABCO mounting solution (Sigma), and examined under a confocal microscope (Fluoview 500, Olympus).

The detection of TBP in oocytes and early embryos was performed 
using the same protocol as for TRF3, except that oocytes and embryos were washed twice in PBS, fixed for $1 \mathrm{~h}$ in $4 \%$ PFA in PBS, and then permeabilized with PBST $2 \times$ for $10 \mathrm{~min}$ at room temperature.

Immunofluorescence of NIH3T3 cells

The distribution of TBP in HeLa cells varies, depending on the methods used for fixation and permeabilization of the cells [29]. We therefore performed immunocytochemical studies using two different procedures. Cells grown on glass slides were washed twice in PBS and treated by one of two protocols: (a) immediate fixation in $4 \%$ PFA in PBS for 10 min and permeabilization in PBST for $2 \times 10 \mathrm{~min}$; or (b) permeabilization in PBST for $10 \mathrm{~min}$ on ice and subsequent fixation in 4\% PFA for $10 \mathrm{~min}$. Cells were then rinsed in PBST and incubated with primary and secondary antibodies diluted in PBST. Distribution of TBP and TRF3 during different cell cycle phases was detected by protocol a, and distribution of TRF3 within the nucleoli was detected by protocol $b$.

\section{Western blot analysis}

Oocytes and embryos were collected in loading buffer [30] and frozen at $-80{ }^{\circ} \mathrm{C}$ until used. Whole-cell extracts from NIH3T3, D3, and ovary were prepared using mammalian protein extraction regent (Pierce) and performed according to the manufacturer's protocol. Samples were boiled for 5 min and loaded onto $12 \%$ polyacrylamide gels. After electrophoresis, proteins were transferred onto a PVDF membrane (Amersham) for $1 \mathrm{~h}$ at $100 \mathrm{~V}$ in a transfer buffer containing $25 \mathrm{mM}$ Tris, $192 \mathrm{mM}$ glycine, and 20\% (v/v) methanol ( $\mathrm{pH} 8.3$ ). Membranes were soaked for $1 \mathrm{~h}$ in blocking solution ( $5 \%$ non-fat milk in TBS) and incubated with the primary antibody in blocking solution overnight at $4{ }^{\circ} \mathrm{C}$ with gentle agitation. Following three washes in TBS containing $0.1 \%$ Tween-20 (TBST), membranes were incubated for $1 \mathrm{~h}$ in peroxidase-conjugated donkey anti-rabbit IgG (Jackson
Immunoresearch) diluted 1:10 000 in blocking solution, and washed as above. Immunoreactive proteins were visualized using ECL Plus reagents (Amersham) according to the manufacturer's instructions.

\section{ChIP analysis}

ChIP analysis was performed essentially as described [31]. Mouse ES cells (D3) were treated with 1\% formaldehyde at room temperature for 10-15 $\mathrm{min}$. The reaction was stopped by the addition of glycine to a final concentration of $125 \mathrm{mM}$. Cells were washed twice in ice-cold PBS, resuspended in M-PER mammalian protein extraction reagent (Pierce) containing a protease inhibitor cocktail (Sigma), and sonicated on ice until the crosslinked chromatin was sheared to an average DNA fragment length of $0.5-1 \mathrm{kbp}$. After centrifugation, soluble crosslinked chromatin was frozen. ChIP extract was diluted 1:10 in immunoprecipitation (IP) buffer (10 mM HEPES-KOH $(\mathrm{pH}$ 7.9), $1 \%$ Triton $\mathrm{X}-100,150 \mathrm{mM} \mathrm{NaCl}$, and protease inhibitors) and incubated with the antibody for $12-14 \mathrm{~h}$ at $4{ }^{\circ} \mathrm{C}$. Immunoprecipitates were recovered by incubation with Protein A agarose for $2 \mathrm{~h}$ at $4{ }^{\circ} \mathrm{C}$ followed by centrifugation. The beads were washed three or four times with IP buffer, three or four times in wash buffer [10 mM Tris- $\mathrm{HCl}$ ( $\mathrm{pH}$ 8.0), $0.25 \mathrm{mM} \mathrm{LiCl,} 0.5 \% \mathrm{NP}-40,0.5 \%$ sodium deoxycholate, $1 \mathrm{mM}$ EDTA (pH 8.0)], and three or four times in Tris-EDTA (TE) ( $\mathrm{pH} 8.0$ ). The material was eluted in $1 \%$ SDS in sodium carbonate, and digested with $250 \mu \mathrm{g} / \mathrm{ml}$ proteinase $\mathrm{K}$ (Roche) for $12 \mathrm{~h}$ at $37^{\circ} \mathrm{C}$. Formaldehyde crosslinks were reversed by heating for $6 \mathrm{~h}$ at $65^{\circ} \mathrm{C}$. The DNA was purified by extraction with phenol, precipitation with ethanol, and analyzed by PCR with primers near the transcription start site. Primer sequences are listed in Table 1. Antibodies used for ChIP analyses were rabbit polyclonal antibodies raised against N-terminal domain of TBP (Santa Cruz Biotechnology) or TRF3 (made by Abgent as described above). Normal rabbit IgG was used as a negative control.

Table 1 Primers used in ChIP assays

\begin{tabular}{|c|c|c|c|}
\hline$\overline{\text { Gene }}$ & Primer sequence & Position & Product size (bp) \\
\hline \multirow[t]{2}{*}{ Oct 4} & S 5'TGGGGTCCCGTCCTAAGGGTTGT 3' & -187 to 85 & 273 \\
\hline & A 5'GTGAGAAGGCGAAGTCTGAAGC3' & & \\
\hline \multirow[t]{2}{*}{ Nanog } & S 5'GGCTGATTTGGTTGGTGTCTTG3' & 5 to 270 & 266 \\
\hline & A 5'CAGGCATTGATGAGGCGTTC3' & & \\
\hline \multirow[t]{2}{*}{ Rpl30 } & S 5'GAACAAACGCCCAGAACCGAAGG3' & -42 to 203 & 246 \\
\hline & A 5'CTCACCAACACCAGCCGCCAAG3' & & \\
\hline \multirow[t]{2}{*}{ Rpl18 } & S 5'CCTGGAATGTCTTCTATGGAGCAA3' & -375 to -191 & 185 \\
\hline & A 5'TCTGTCGTCGTACCGTTCTTGTG3' & & \\
\hline \multirow[t]{2}{*}{$A c t b$} & S 5'GGATCACTCAGAACGGACACC3' & 143 to 343 & 201 \\
\hline & A 5'GGCTCATCAAATGCCCACA3' & & \\
\hline \multirow[t]{2}{*}{ Tubb2 } & S 5'CAGGTGGACACTGAGGGGAAAC3' & -236 to 46 & 283 \\
\hline & A 5'GA AGGGGTGGAGGCTCTTGC3' & & \\
\hline \multirow[t]{2}{*}{ DSS in $A c t b$} & S 5'AGCCTTCCTTCTTGGGTAA3' & 2367 to 2659 & 293 \\
\hline & A 5'CCTTGATCTTCATGGTGC3' & & \\
\hline \multirow[t]{2}{*}{ DSS in Tubb2 } & S 5'ACCTTCATTGGCAACAGCAC3' & 3335 to 3673 & 339 \\
\hline & A 5'ACAGAGGCAAACTGAGCACC3' & & \\
\hline
\end{tabular}


A

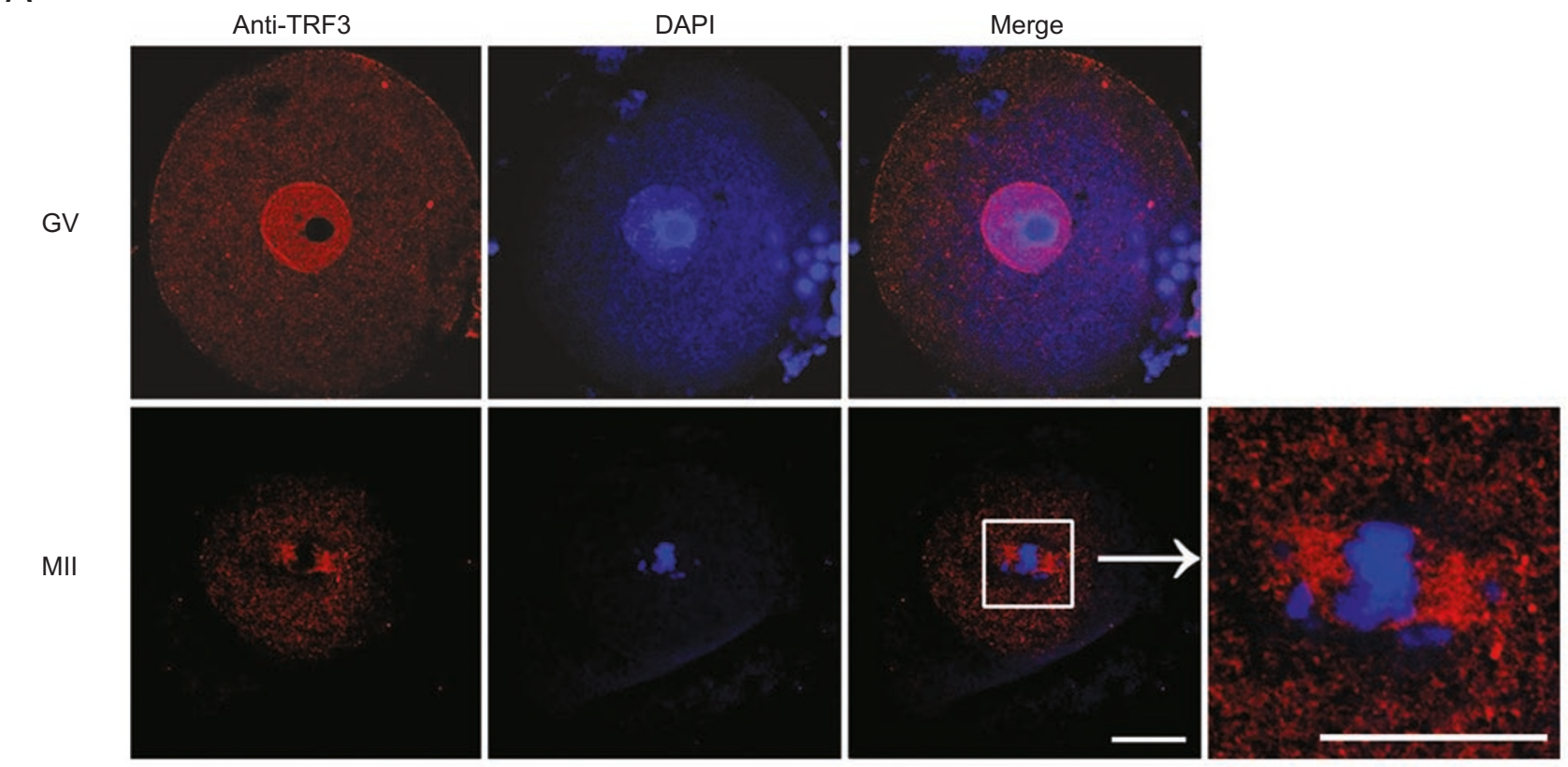

B

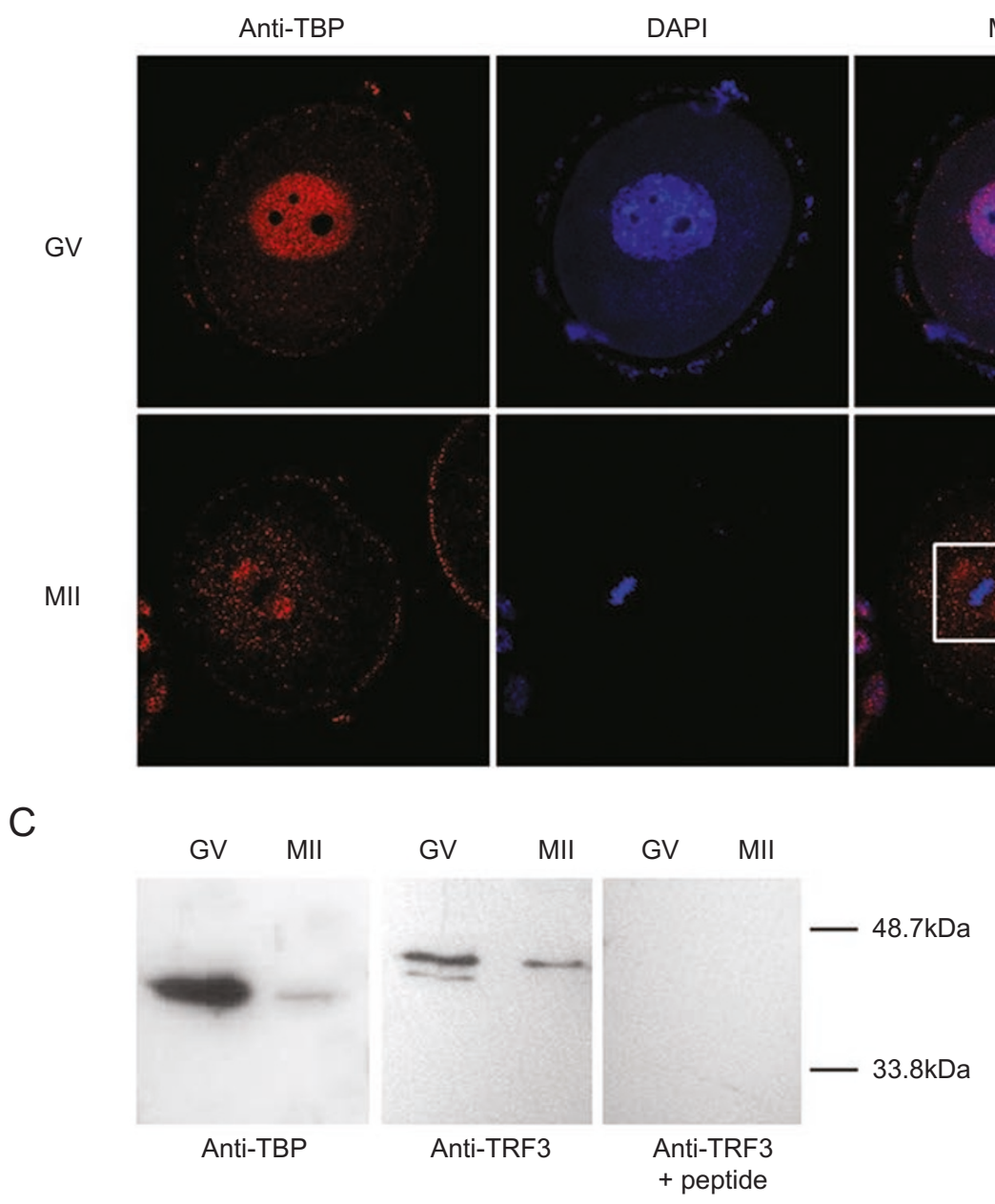

Figure 1 Expression of TBP and TRF3 in the oocytes and MII eggs. Oocytes and MII eggs were labeled with anti-TRF3 (A) and anti-TBP (B) antibodies. In (A and $\mathbf{B}$ ): upper panels, GV-stage oocytes; lower panels, MII eggs. The arrow indicates a higher magnification view of the square. (C) GV-stage oocytes and MII eggs (200 per lane) were immunoblotted using antiTBP antibody, anti-TRF3 antibody, or anti-TRF3 antibody preincubated with the immunogenic peptide. Mw markers are indicated to the right of the blots. Scale bar $=20 \mu \mathrm{m}$. 
A

6hPF
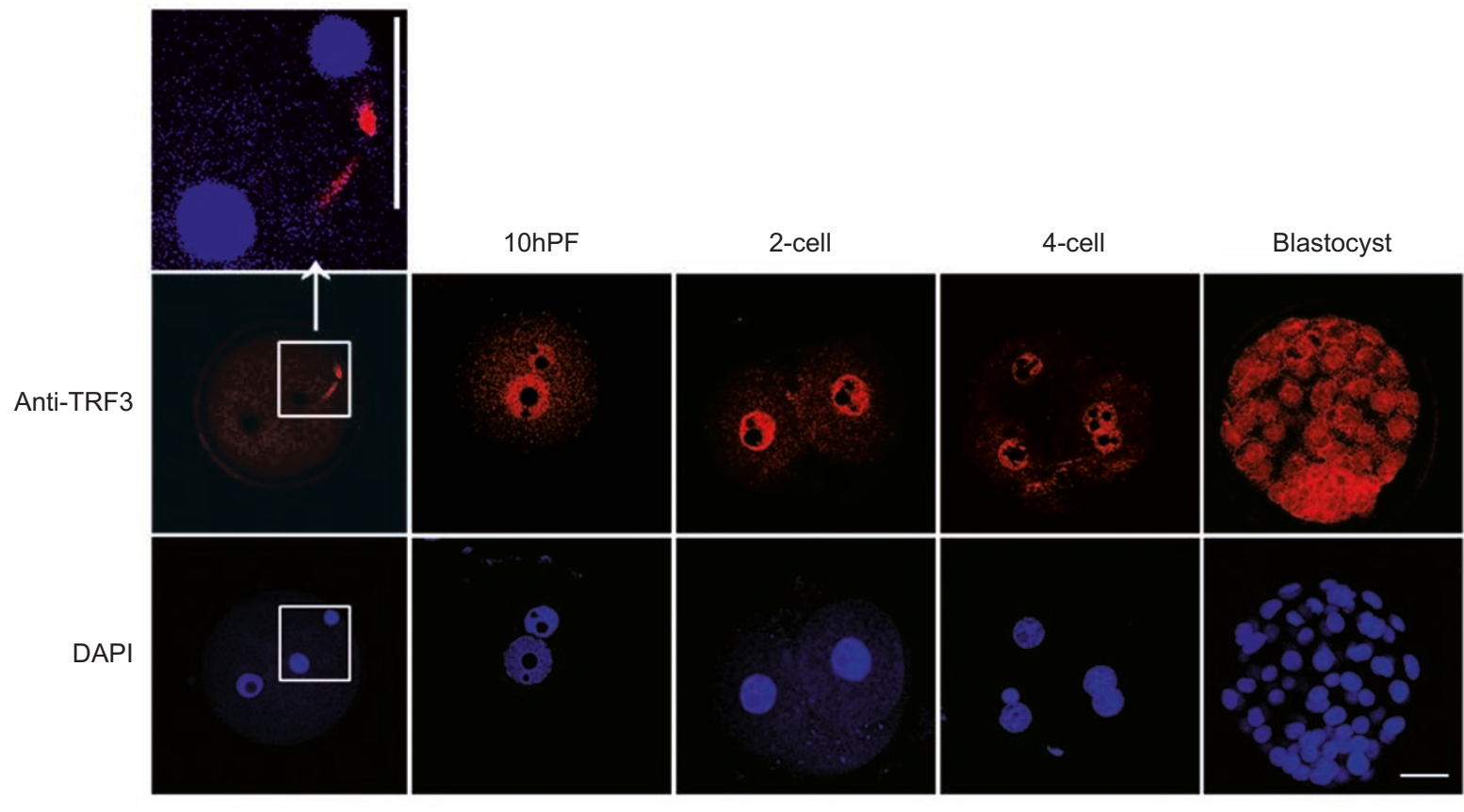

B

6hPF

10hPF

2-cell

4-cell

Blastocyst

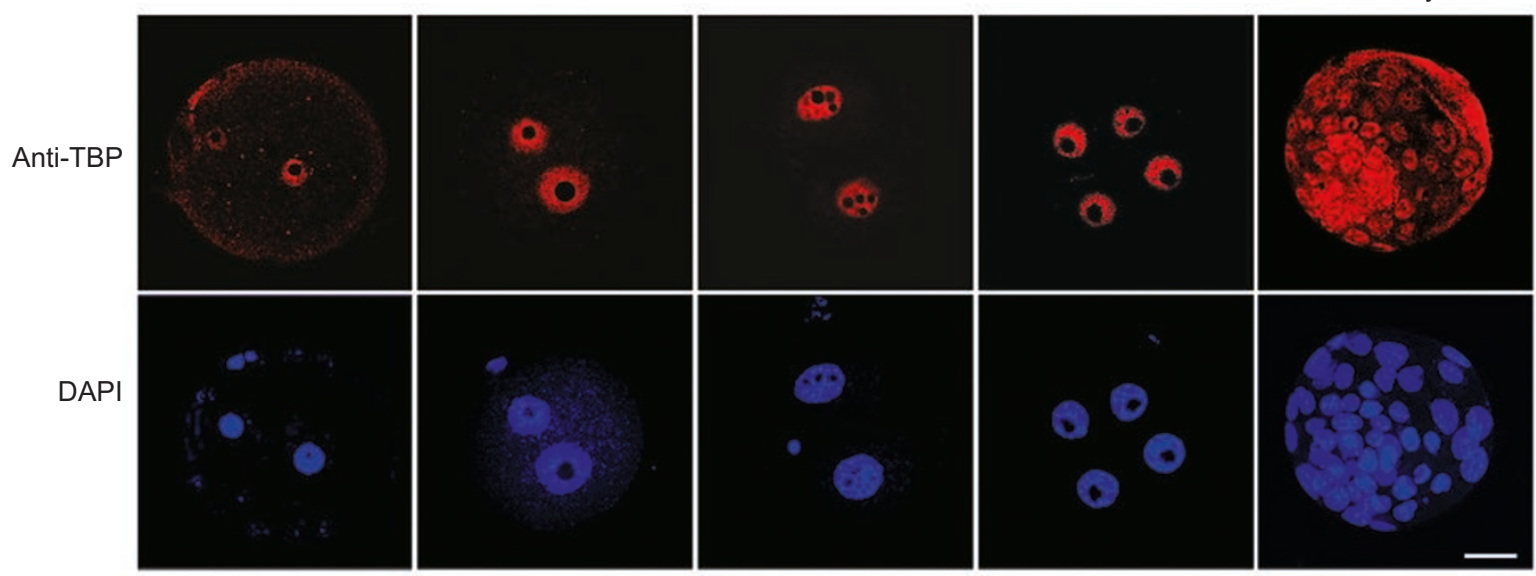

C

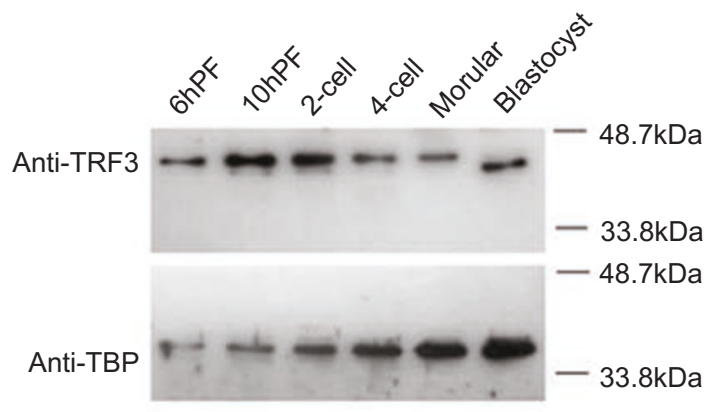

D

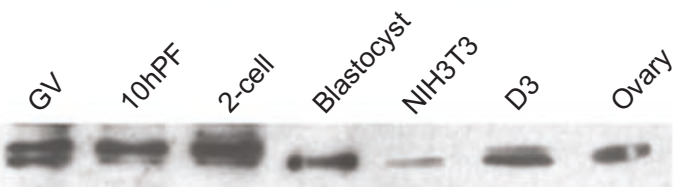




\section{Results}

Expression of TBP and TRF 3 during meiotic maturation

To determine TRF3 expression just before and at the onset of development, oocytes at the GV and MII stages were labeled with an affinity-purified antibody against the N-terminal domain of mouse TRF3 and examined by confocal microscopy. In the GV oocytes, labeling was concentrated in the GV (Figure 1A, upper panels). In the MII oocyte, TRF3 appeared as two concentrated dots flanking the condensed chromosomes (Figure 1A, lower panels). No obvious signal was detected on the condensed chromosomes. The subcellular distribution of TBP was similar to TRF3 in both the GV oocytes and MII eggs (Figure 1B). The TRF3 labeling was specific, since incubation of the antibody with immunogenic peptides abolished nuclear fluorescence in the immunochemical assay (data not shown) and Western blot signals (Figure 1C, right panel).

Immunoblot analysis with the TRF3 antibody detected a major band of approximately $41 \mathrm{kDa}$ and a minor band with a slightly lower molecular weight $(\mathrm{mw})$. While the GV oocytes contained both variants, the MII eggs mainly contained the higher mw TRF3. The amount of TRF3 protein decreased by $>70 \%$ in the MII oocytes compared to the GV oocytes (Figure 1A and 1C (middle panel)). TBP was detected as a heavy, single band in the GV oocytes. The amount of TBP decreased by $>90 \%$ by the MII stage (Figure 1B and 1C (left panel)).

\section{Expression of TBP and TRF3 during early embryogen- esis}

We next examined temporal changes in TRF3 and TBP expression in preimplantation embryos (Figure 2). At $6 \mathrm{~h}$ postfertilization (PF), TRF3 appeared as two separate patches outside the two pronuclei (between the female pronucleus and the polar body) (Figure 2A). Only at $10 \mathrm{~h}$ PF were obvious TRF3 signals detected in the pronuclei of most one-cell embryos (11/15). TRF3 was detected in the nuclei of embryos at the two-cell $(45 / 50)$, four-cell (23/32), and blastocyst stages (27/38). At the blastocyst stage, TRF3 was observed in cells of both the inner cell mass and trophoectoderm. TBP was readily detected in the pronuclei of most embryos $6 \mathrm{~h} \mathrm{PF} \mathrm{(12/15),} \mathrm{and} \mathrm{expression}$ was maintained in all embryos from $10 \mathrm{~h} \mathrm{PF}$ to the blastocyst stage (Figure 2B). Both the TBP and TRF3 signals were concentrated in the pronuclei of the one-cell embryo $10 \mathrm{~h} \mathrm{PF}$ and in the nuclei of the two-cell-, four-cell-, and blastocyst-stage embryos.

To examine changes in the amounts of TRF 3 and TBP during early embryogenesis, embryos at different preimplantation stages were collected and analyzed by Western blot. The MII oocytes and early embryos up to the morular stage mainly contained the higher mw variant of TRF3 (Figure 2C and 2D). However, the lower mw variant of TRF3 became the dominant form in blastocysts and mouse ES cells (the D3 line), as well as the NIH3T3 cell line and ovary. Therefore, while the GV-stage oocyte contained both TRF3 variants, the MII egg and early embryos up to the morular stage mainly contained the high mw variant, whereas blastocysts and somatic cells and tissues at later developmental stages mainly contained the lower mw variant. The functional implication and origin of these variants, such as whether they are derived from alternative splicing or post-translational modification, has not yet been determined.

The level of TBP was relatively low in the one-cell embryos and increased gradually and steadily as embryos developed through the preimplantation stages (Figure 2C, lower panels). Similar to TBP, the total amount of TRF3 in the cell increased after fertilization (Figure 2C, upper panels). However, the total amount of TRF3 increased drastically in the one-cell embryo at $10 \mathrm{~h} \mathrm{PF}$, reached a peak in two-cell embryos, and decreased slightly thereafter. The sharp increase in the nuclear concentration of TRF3 observed at the one- and two-cell stages coincides with the timing of ZGA.

\section{TRF3 enters the newly formed nucleus later than TBP}

We have demonstrated that TRF3 is co-expressed with TBP in maturing oocytes and in early mouse embryos (Fig-

\footnotetext{
Figure 2 Expression of TBP and TRF3 in preimplantation embryos, cell lines, and tissue. (A) Embryos labeled with anti-TRF3. (B) Embryos labeled with anti-TBP. In (A and B): upper panels, immunofluorescent labeling; bottom panels, the same embryos counterstained with DAPI. The arrow indicates a merged and higher magnification view of the two squares. The developmental stages are indicated above each lane. It should be noted that the position of the pronuclei in the one-cell embryos may be affected by the staining procedures, and therefore the distance between the male and female pronuclei shown in the figure may not represent the distance seen under the light microscope before immunolabeling. (C) Early embryos at different stages (200 per lane) were immunoblotted using purified anti-TRF3 (upper panel) or anti-TBP (lower panel) antibodies. (D) TRF3 protein in maturing oocytes, preimplantation embryos, tissue (ovary), and cell lines (NIH3T3 and D3). For oocytes and embryos, 200 per lane; for ovary, NIH3T3 cells, and D3 cells, $10 \mu \mathrm{g}$ total protein per lane. Scale bar $=20 \mu \mathrm{m}$.
} 
A

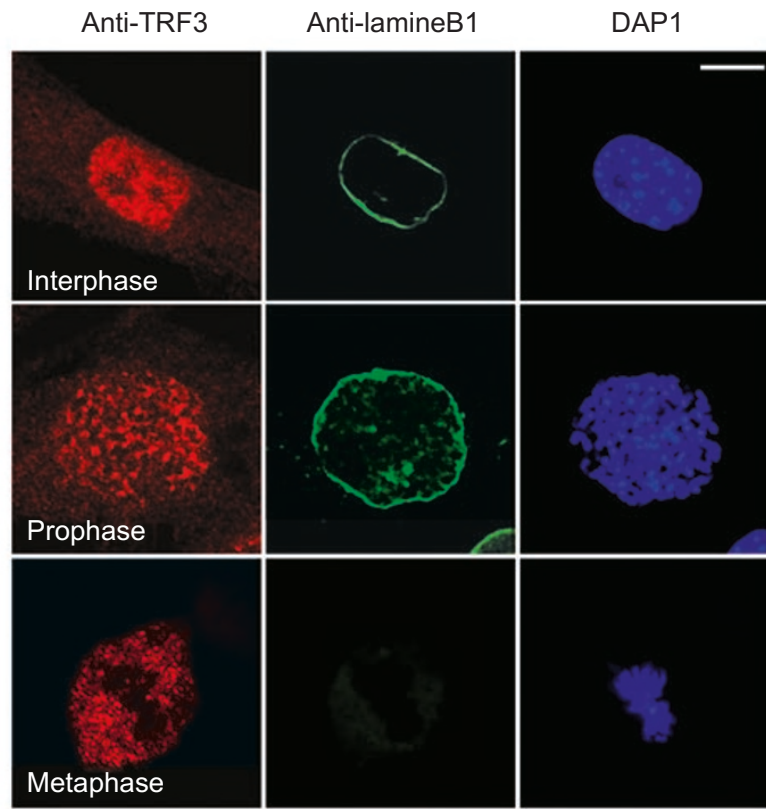

C

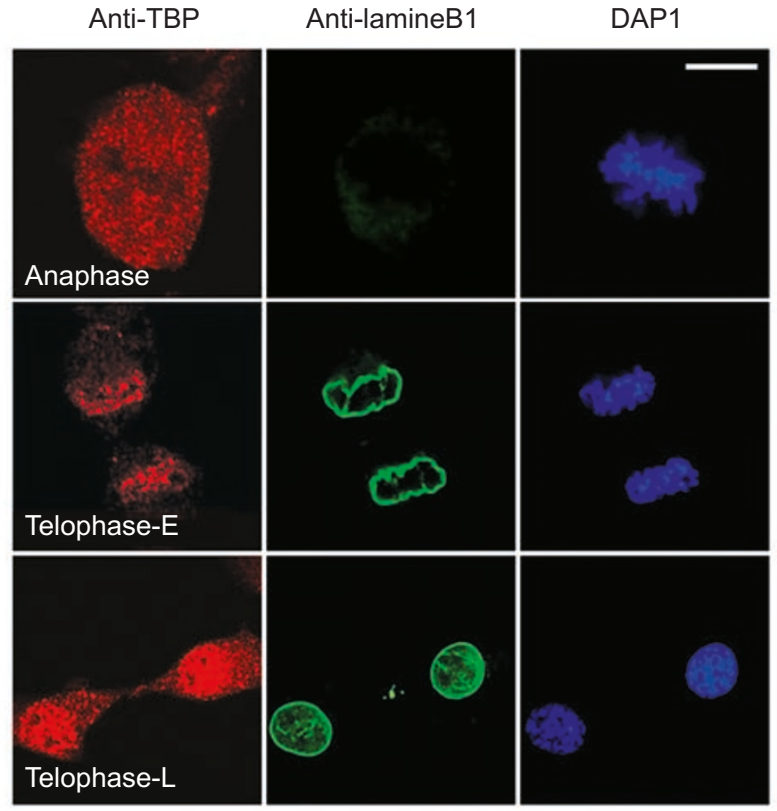

B

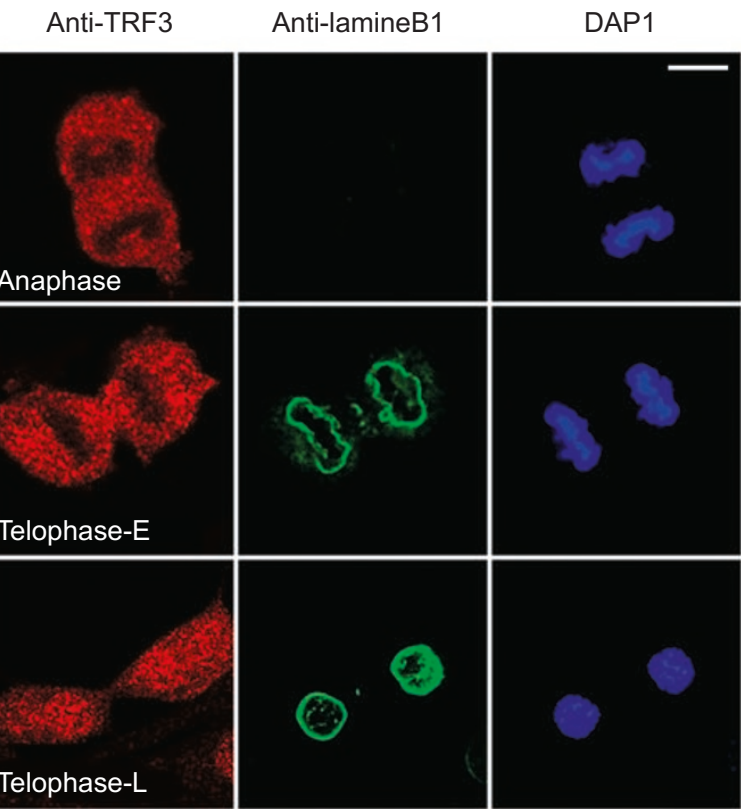

D

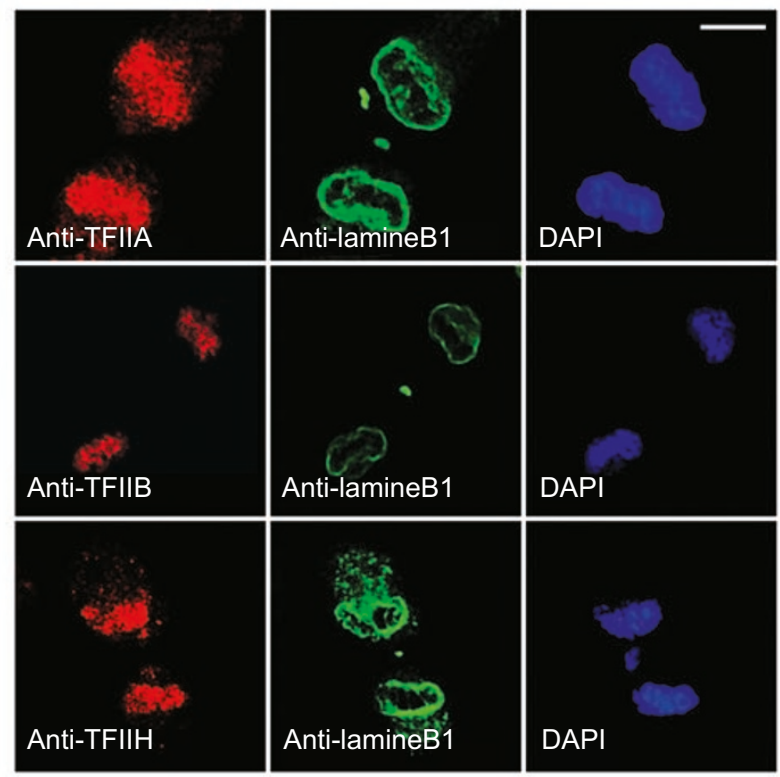

Figure 3 Localization of TBP and TRF3 in NIH3T3 cells in different phases of the mitosis cycle. (A and B) Double labeling of fixed, permeabilized cells with antibodies against TRF3 (red) and lamin B1 (green) and counterstaining with DAPI (blue). (C and D) Double labeling with antibodies against TBP, TFIIA, TFIIB, or TFIIH (red), and lamin B1 (green). Scale bar=10 $\mu \mathrm{m}$.

ures 1 and 2). However, although TBP was detected in the pronuclei soon after their formation (at approximately $6 \mathrm{~h}$ $\mathrm{PF}), \mathrm{TRF} 3$ was not present in the pronuclei until approximately $10 \mathrm{~h}$ after PF, several hours later than TBP (Figure $2 \mathrm{~A}$ and $2 \mathrm{~B}$ ). The delay of TRF3 in entering the newly formed pronucleus in the early embryo may be analogous to the observation in HeLa cells where TRF3 was delayed in entering the nucleus after mitosis [20].

To confirm that TRF3 was delayed in entering the nucleus in somatic cells after mitosis, we double-labeled NIH3T3 cells with an antibody against lamin B1, a component of the nuclear membrane, and the anti-TRF3 antibody. 
As controls, we also labeled cells with antibodies against TBP and other components of preinitiation complex, including TFIIA, TFIIB, and TFIIH. The results showed that during interphase, TRF3 was in the nucleus. As the cell entered mitosis, TRF3 was found in the cytoplasm but not on chromosomes (Figure $3 \mathrm{~A}$ and $3 \mathrm{~B}$ ), suggesting that most TRF3 was excluded from the condensed chromatin from prophase to anaphase. In these aspects, TRF3 behaves very similar to TBP (Figure $3 \mathrm{C}$ and Kieffer-Kwon et al. [32]). In early telophase (Telophase-E), when the nuclear envelope, highlighted by the antibody to lamin B1, had just formed around the daughter nuclei, TBP was already concentrated in the nuclei (Figure 3C), whereas TRF3 was still predominantly in the cytoplasm (Figure 3B). Only at the late telophase did TRF3 become dominant in the daughter nuclei (Figure 3B). Similar to TBP, TFIIA, TFIIB, and TFIIH were also predominantly localized in the newly formed daughter nucleus at Telophase-E after mitosis (Figure 3D).
Our results indicated that most TRF3, as well as several other members of the general transcription machinery, was ejected from the condensing or condensed chromatin during mitosis and re-imported into the nucleus on exiting the cell cycle. TRF3 and TBP behaved similarly in this aspect. However, TRF3 was re-imported back into the nucleus slightly later than other general transcription factors (GTFs). This sequential nuclear import of TBP followed by TRF3 suggests that they have different roles in the transcriptional reactivation program after mitosis.

\section{TRF3 is recruited to gene promoters in mouse ES cells}

TRF3 is a GTF and should be recruited to promoters in order to function in early development. Since it was difficult to collect enough preimplantation-stage mouse embryos for biochemical analyses, we used mouse ES cells instead. ES cells are originally isolated from blastocysts and regarded as an excellent model system for the investigation of cellular events accompanying embryonic development [33].

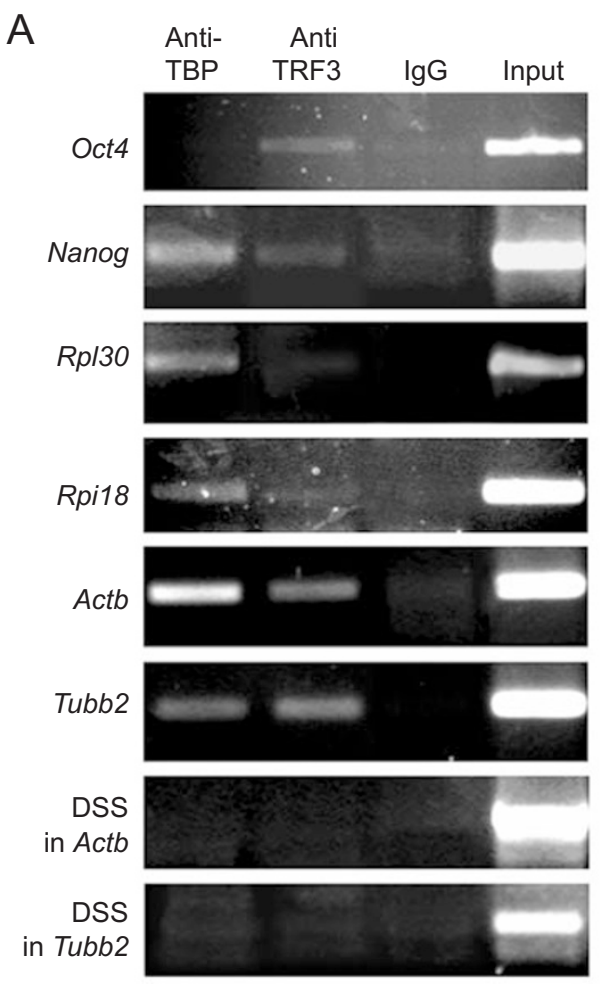

B
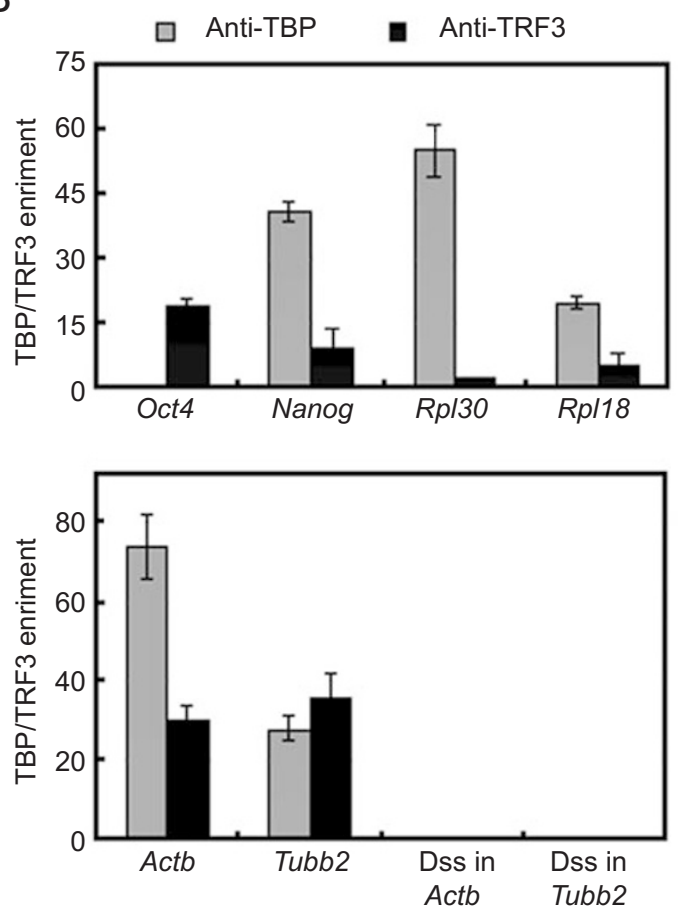

Figure 4 Recruitment of TBP and TRF3 to the promoters as assayed by ChIP. Chromatin fragments immunoprecipitated by anti-TBP or anti-TRF3 antibodies or normal rabbit IgG were amplified by PCR with primer pairs flanking or near the transcription start site of Oct 4, Nanog, Rpl30, Rpl18, Actb, and Tubb2 genes (Table 1). DSS in Actb and Tubb2 genes were also amplified as negative controls. PCR products were resolved by agarose-gel electrophoresis (A). The IgG control defines the non-specific binding of TBP/TRF3 protein to IgG. The intensity of the bands from the input and IgG was set as 100 and 0 , respectively. The intensity of other bands was calculated and shown as histograms in (B). Bars represent the mean intensity of the bands obtained from three independent experiments. Error bars show the standard error of the mean. 
A

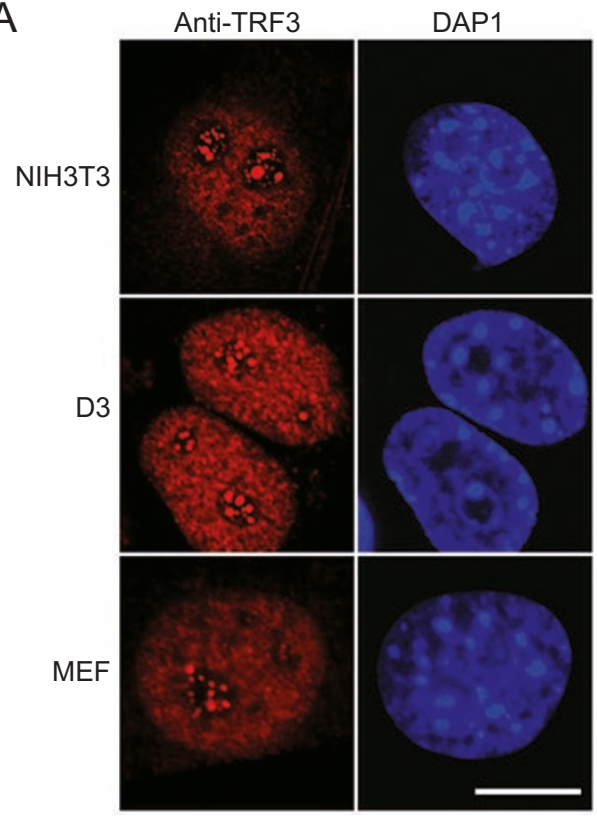

B
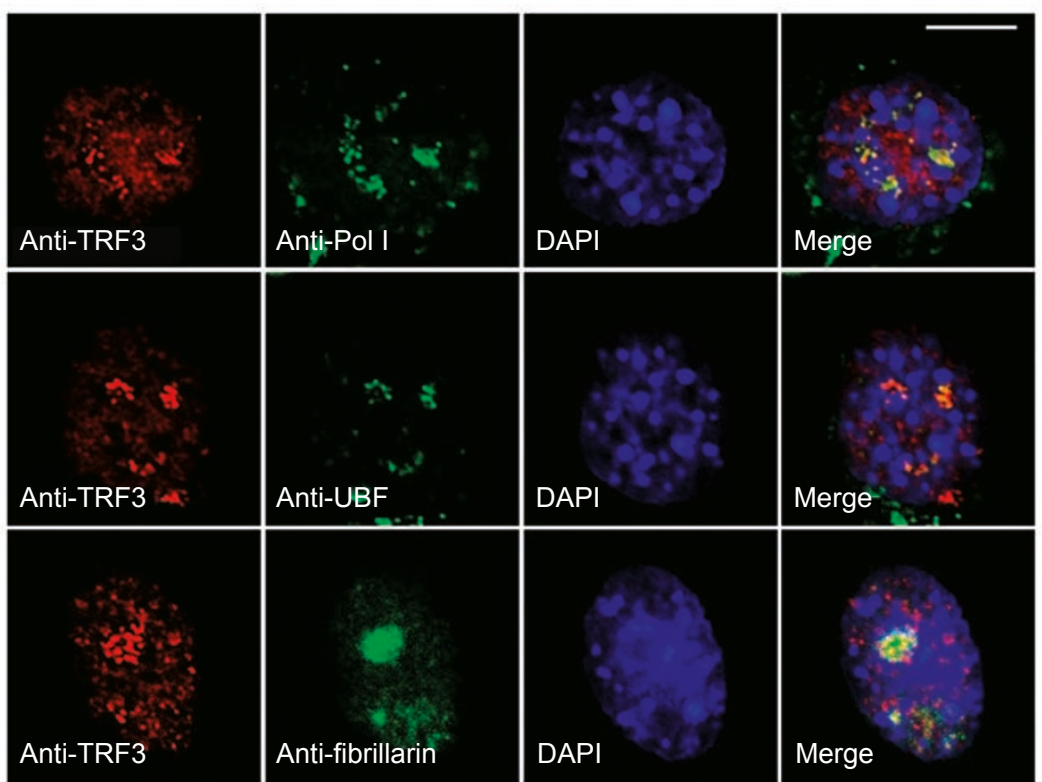

Figure 5 Localization of TRF3 within the nucleoli in cell lines. (A) Permeabilized, fixed cells were labeled with antibodies against TRF3. (B) NIH3T3 cells were double-labeled with antibodies against TRF3 (red) and Pol I, UBF, or fibrillarin (green) as indicated.

ChIP assays were performed using anti-TRF3 or antiTBP antibodies to examine the promoter-binding status of the two factors. Both TATA-box containing ( $R p l$ 18, Actb, and Tubb 2) and TATA-less (Oct 4, Nanog, and Rpl30) genes were chosen as the targets of our ChIP assays. Among them, Oct 4 and Nanog genes are undifferentiated cells specific genes, whereas Rpl30, Rpl18, Actb, and Tubb 2 are housekeeping genes. As shown in Figure 4, TBP recruitment to Nanog, Rpl30, Rpl18, Actb, and Tubb 2 promoters was high (20-74\% of the input), whereas TBP recruitment to Oct 4 promoter was undetectable. TRF3 recruitment to $A c t b$ and $T u b b 2$ promoters was high (above $30 \%$ of the input) and its recruitment to Oct 4 and Nanog promoters was evident (18\% and $9 \%$, respectively), whereas TRF3 recruitment to $R p l 30$ and $R p l 18$ promoters was much lower (under $5 \%$ of the input). The recruitment of TBP and TRF3 to these promoters is specific since neither TBP nor TRF3 bound to the downstream sequence (DSS) in Actb or Tubb 2 genes. Each of these experiments was repeated at least three times and results were consistent. Data from the ChIP assay therefore suggested that different gene promoters may recruit TBP or TRF3 selectively. While some promoters use either TRF3 or TBP, others require both of them. Similar to TBP, TRF 3 bound to not only TATA-containing promoters (Actb and Tubb 2) but also to TATA-less promoters (Oct 4 and Nanog). Data from biochemical analyses, consistent with immunocytochemical results, indicated that TRF3 may participate in transcription during early embryogenesis.

\section{Co-localization of TRF3 with Pol I and UBF in the nucleoli}

Using a modified immunocytochemistry protocol, TRF3-reactive speckles were seen within the nucleolus in mouse ES, MEF, and NIH3T3 cells (Figure 5A).

To examine whether TRF3 participated in the transcription complex for Pol I transcription, we performed doublelabeling assays in NIH3T3 cells using antibodies against Pol I, the Pol I transcriptional activator UBF, and fibrillarin, each in combination with the anti-TRF3 antibody. Both Pol I and UBF are components of the Pol I transcription complex and associated with rDNA loci within the nucleolus $[29,32,34]$. Fibrillarin is a structural component of the nucleolus and appears as homogeneous distribution [32]. We found that Pol I, UBF, and TRF3 appeared as discrete speckles within the nucleolus, whereas fibrillarin was more homogeneously distributed within the nucleolus. TRF3 co-localized with UBF and Pol I but not fibrillarin (Figure 5B). These data suggest that TRF3 may be a component of the Pol I transcription complex and may have a role in Pol I transcription. However, using whatever method, we could not detect such discrete TRF3-reactive speckles within the nucleolus in oocytes and preimplantation mouse embryos where TRF3 was distributed within the nucleoplasm as shown in Figures 1A and 2A. 


\section{Discussion}

Our results have shown that TRF3 is co-expressed with TBP in both the maturing oocytes and embryos, and selectively binds to some gene promoters in ES cells. In addition, TRF3 proceeds through most phases of the cell cycle at a pace parallel to TBP, except Telophase-E, when TRF3 was slightly delayed in entering the nucleus. Based on the fact that (1) TRF3 has a conserved C-terminal region and a DNA-binding domain virtually identical to TBP [20, 21], (2) TRF3 can mediate transcription initiation in both HeLa cell extract and Xenopus egg extract [20, 22], (3) TRF3 is co-expressed with TBP during early development, and its dynamic change correlates well with transcriptional activity (Figures 1 and 2), (4) both factors proceed through most cell cycle phases at a similar pace (Figure 3), and (5) TRF3 binds to the promoter(s) of genes that are expressed in early embryogenesis (Figure 4), we suggest that TRF3 may play a role in early embryonic development. Our results support the proposal that TRF3 is a possible candidate for TBP-independent transcription in the $T B P^{-/-}$cells $[20,22]$. However, more extensive biochemical analyses are needed to confirm the hypothesis.

Dynamic changes in the amounts of both TBP and TRF3 in maturing oocytes and early embryos appeared to be correlated with transcriptional activity during these periods. In the mouse, transcription is active in the GV oocytes and ceases by the MII stage [35]. The transcriptionally silent state persists in the one-cell embryo after fertilization until the minor ZGA at approximately $10 \mathrm{~h}$ PF [24-26]. Concomitantly, TBP and TRF3 are found in the $\mathrm{GV}$ of the oocyte and their amounts decrease sharply, by $90 \%$ and $70 \%$, respectively, by the MII stage (Figure 1). Several hours before ZGA, the amounts of both factors begin to increase and reach high levels when ZGA occurs (Figure 2). Since proteins detected by Western blot were from whole embryos, a decrease in the amount of TBP and TRF3 after the GV stage and their subsequent recovery in the one-cell embryo cannot be explained by translocation of these factors within the cells. The drastic reduction in both factors after the GV stage likely represents a loss of transcription factors, possibly through degradation. Loss of transcription factors and the transcriptional silence that occurs after the GV stage may be intrinsically related. The increase in TBP and TRF3 in the one-cell embryo before ZGA may result from increased synthesis of transcription factors. In the mouse, transcription is silent at $6 \mathrm{~h} \mathrm{PF}$, minor ZGA begins at $10 \mathrm{~h} \mathrm{PF}$, and major ZGA begins at the late two-cell stage. Both TBP and TRF3 were imported into the pronuclei before ZGA. A significant increase in the amount of TBP and TRF3 just before ZGA suggests a role for both factors in the activation of zygotic transcription.
Importation of TBP and TRF3 back to the nucleus follows a temporal order, which may relate to differential functions of the two factors.

Recruitment of TBP and TRF3 to promoters has been studied by ChIP in Xenopus embryos [22]. At gastrula and tailbud, both TBP and TBP2 were observed on the H2B.1, $M y o D$, and $I d 3$ promoters. TRF3 binds to $E F 1 \alpha$ promoter at high levels in gastrula and tailbud stages and then decreases, whereas TBP recruitment is undetectable in gastrula and tailbud stages but increases dramatically in tadpoles. This finding suggests the gene-selective roles of TBP and TRF3 in gene expression at special developmental stage. Our results showed that TBP and TRF3 are selectively recruited to gene promoters in mouse ES cells (Figure 4), which give another evidence for their specialized role in gene expression. It should be mentioned that neither TBP nor TRF3 was detected on promoters of some genes that are expressed in ES cells (data not shown), which indicates other TBPindependent transcription mechanism(s) exists.

During mitosis, both TRF3 and TBP were detected in the cytoplasm, but not on the condensing or condensed chromosomes by immunocytochemistry (Figure 3A-3C). This observation is in agreement with reports by Kieffer-Kwon et al. [32] and Segil et al. [36]. However, in alternative assays such as GFP-coupled TBP [37] or ChIP assays [31], a substantial amount of TBP was associated with condensed chromosomes during mitosis. Several reasons may account for this discrepancy. First, structural changes or epigenetic modification of chromatin during mitosis may block the access of antibodies to TBP, TRF3, or other chromatin-associated factors, whereas such spatial obstacles would not prevent visualization of chromatinassociated factors in biochemical assays or when using GFP-coupled TBP. Second, using immunocytochemistry, a uniform staining was seen over the mitotic cytoplasm, which was significantly higher than that seen in the control (Figure 3A-3C). This suggests that a significant amount of TRF3 and TBP were dissociated from the chromatin and distributed in the cytoplasm. Since the antibody may not be able to access chromatin-associated factors on the chromosome, we saw more signals in the cytoplasm than in the chromosome. In contrast, in the GFP-coupled TBP assay, the condensing chromatin might increase the local concentration of GFP-coupled TBP, leading to a chromatin with more fluorescent signal than the cytoplasm [37]. Our data support the idea that while a small proportion of the cellular TBP and TRF3 remains associated with specific promoter loci, a substantial amount of transcription factors dissociate from the chromatin during mitosis [32].

TBP is required for Pol I transcription [1]. In HeLa cells, TBP has been found to co-localize with UBF and Pol I at rDNA loci, which displays as discrete speckles within the 
nucleoli [29]. TRF2 also locates in the nucleoli in HeLa cells, but displays more homogeneous distribution and is not co-localized with UBF [32]. The author therefore concluded that TRF2 is only stored in the nucleoli but is not required for Pol I transcription. In our double immunocytochemical analyses, TRF3 was found to locate in the nucleoli and was co-localized with Pol I and UBF (Figure 5 ). These results suggest that, in addition to playing a role in Pol II transcription [21, 22], TRF3 may also be a component of the rRNA transcription machinery and may be involved in Pol I transcription. Interestingly, we could not detect TRF3 in the nucleoli of early mouse embryos using the same methods, possibly because the nucleoli and the machinery for Pol I transcription are organized differently in early embryos and in somatic and ES cells. Therefore, TRF3 may be involved in Pol I transcription in tissues or cells at later stages but not in early embryos. This observation is consistent with the previous finding that TRF3 does not substitute for TBP function in Pol I- and Pol III-mediated transcription in $\mathrm{TBP}^{-/-}$early mouse embryos [17].

\section{Acknowledgments}

We thank Profs Qing Zhang Yang and Feng Ying Xing for technical assistance in oocytes and early embryos collection; You Ming Zhu for providing culture medium and Feng Sun for support in immunochemical staining. This study was supported by grants from National Basic Research Program of China (973 Program) (Nos. $001 \mathrm{CB} 509903$ and 001CB509904), Hi-Tech Research and Development Program of China (863 Program) (Nos. 2001AA216121 and 2004AA205010), National Natural Science Foundation of China (No. 30040003), Science and Technology Committee of Shanghai Municipality (Nos. 99DJ14002, 00DJ1 4033, 01DJ14003, and 03DJ14017), Chinese Academy of Science (No. KSCX-2-3-08), and Shanghai Municipal Education Commission and Shanghai Jiao Tong University, School of Medicine.

\section{References}

1 Burley SK, Roeder RG. Biochemistry and structural biology of transcription factor IID (TFIID). Annu Rev Biochem 1996; 65:769-799.

2 Comai L, Tanese N, Tjian R. The TATA-binding protein and associated factors are integral components of the RNA polymerase I transcription factor, SL1. Cell 1992; 68:965-976.

3 Chiang CM, Ge H, Wang Z, Hoffmann A, Roeder RG. Unique TATA-binding protein-containing complexes and cofactors involved in transcription by RNA polymerases II and III. EMBO J 1993; 12:2749-2762.

4 Hernandez N. TBP, a universal eukaryotic transcription factor? Genes Dev 1993; 7:1291-1308.

5 Crowley TE, Hoey T, Liu JK, et al. A new factor related to
TATA-binding protein has highly restricted expression patterns in Drosophila. Nature 1993; 361:557-561.

6 Dantonel JC, Wurtz JM, Poch O, Moras D, Tora L. The TBP-like factor, an alternative transcription factor in metazoa? Trends Biochem Sci 1999; 24:335-339.

7 Ohbayashi T, Kishimoto T, Makino Y, et al. Isolation of cDNA, chromosome mapping, and expression of the human TBP-like protein. Biochem Biophys Res Commun 1999; 255:137-142.

8 Moore PA, Ozer J, Salunek M, et al. A human TATA binding protein-related protein with altered DNA binding specificity inhibits transcription from multiple promoters and activators. Mol Cell Biol 1999; 19:7610-7620.

9 Rabenstein MD, Zhou S, Lis JT, Tjian R. TATA box-binding protein (TBP)-related factor 2 (TRF2), a third member of the TBP family. Proc Natl Acad Sci USA 1999; 96:4791-4796.

10 Teichmann M, Wang Z, Martinez E, et al. Human TATA-binding protein-related factor-2 (hTRF2) stably associates with hTFIIA in HeLa cells. Proc Natl Acad Sci USA 1999; 96:13720-13725.

11 Berk A J. TBP-like factors come into focus. Cell 2000; 103:58 .

12 Dantonel JC, Quintin S, Lakatos L, Labouesse M, Tora L. TBP-like factor is required for embryonic RNA polymerase II transcription in C. elegans. Mol Cell 2000; 6:715-722.

13 Kaltenbach L, Horner MA, Rothman JH, Mango SE. The TBPlike factor CeTLF is required to activate RNA polymerase II transcription during C. elegans embryogenesis. Mol Cell 2000; 6:705-713.

14 Veenstra GJ, Weeks DL, Wolffe AP. Distinct roles for TBP and TBP-like factor in early embryonic gene transcription in Xenopus. Science 2000; 290:2312-2315.

15 Muller F, Lakatos L, Dantonel J, Strahle U, Tora L. TBP is not universally required for zygotic RNA polymerase II transcription in zebrafish. Curr Biol 2001; 11:282-287.

16 Veenstra GJ, Wolffe AP. Gene-selective developmental roles of general transcription factors. Trends Biochem Sci 2001; 26:665671.

17 Martianov I, Viville S, Davidson I. RNA polymerase II transcription in murine cells lacking the TATA binding protein. Science 2002; 298:1036-1039.

18 Martianov I, Fimia GM, Dierich A, et al. Late arrest of spermiogenesis and germ cell apoptosis in mice lacking the TBP-like TLF/TRF2 gene. Mol Cell 2001; 7:509-515.

19 Zhang D, Penttila TL, Morris PL, et al. Spermiogenesis deficiency in mice lacking theTrf2 gene. Science 2001; 292:11531155.

20 Persengiev SP, Zhu X, Dixit BL, et al. TRF3, a TATA-boxbinding protein-related factor, is vertebrate specific and widely expressed. Proc Natl Acad Sci USA 2003; 100:14887-14891.

21 Ba'rtfaii R, Balduf C, Hilton T, et al. TBP2, a vertebrate-specific member of the TBP family, is required in embryonic development of zebrafish. Curr Biol 2004; 14:593-598.

22 Jallow Z, Jacobi UG, Weeks DL, Dawid IB, Veenstra GJ. Specialized and redundant roles of TBP and a vertebrate-specific TBP paralog in embryonic gene regulation in Xenopus. Proc Natl Acad Sci USA 2004; 101:13525-13530.

23 Worrad DM, Ram PT, Schultz RM. Regulation of gene expression in the mouse oocyte and early preimplantation embryo, developmental changes in Spl and TATA box-binding protein, TBP. Development 1994; 120:2347-2357. 
24 Schultz RM. The molecular foundations of the maternal to zygotic transition in the preimplantation embryo. Hum Reprod Update 2002; 8:323-331.

25 Ram PT, Schultz RM. Reporter gene expression in G2 of the 1-cell mouse embryo. Dev Biol 1993; 156:552-556.

26 Aoki F, Worrad DM, Schultz RM. Regulation of transcriptional activity during the first and second cell cycles in the preimplantation mouse embryo. Biol Reprod 1997; 181:296-307.

27 Schultz RM, Montgomery RR, Belanoff JR. Regulation of mouse oocyte maturation, implication of a decrease in oocyte cAMP and protein dephosphorylation in commitment to resume meiosis. Dev Biol 1983; 97:264-273.

28 Chatot CL, Ziomek CA, Bavister BD, Lewis JL, Torres I. An improved culture medium supports development of random-bred 1-cell mouse embryos in vitro. J Reprod Fertil 1989; 86:679688.

29 Jordan P, Mannervik M, Tora L, Carmo-Fonseca M. In vivo evidence that TATA-binding protein/SL1 colocalizes with UBF and RNA polymerase I when rRNA synthesis is either active or inactive. J Cell Biol 1996; 133:225-234.

30 Harlow E, Lane D. Antibodies, A Laboratory Manual. New York: Cold Spring Harbour Press, 1988.
31 Christova R, Oelgeschlager T. Association of human TFIID promoter complexes with silenced mitotic chromatin in vivo. Nat Cell Biol 2002; 4:79-82.

32 Kieffer-Kwon P, Martianov I, Davidson I. Cell-specific nucleolar localization of TBP-related factor 2. Mol Biol Cell 2004; 15:4356-4368.

33 Rathjen J, Rathjen PD. Mouse ES cells: experimental exploitation of pluripotent differentiation potential. Curr Opin Genet Dev 2001; 11:587-594.

34 Zatsepina OV, Voit R, Grummt I, et al. The RNA polymerase I-specific transcription initiation factor UBF is associated with transcriptionally active and inactive ribosomal genes. Chromosoma 1993; 102:599-611.

35 Moore G PM, Lintern-Moore S. Transcription of the mouse oocyte genome. Biol Reprod 1978; 17:865-870.

36 Segil N, Guermah M, Hoffmann A, Roeder RG, Heintz N. Mitotic regulation of TFIID, inhibition of activator-dependent transcription and changes in subcellular localization. Genes Dev 1996; 10:2389-2400.

37 Chen D, Hinkley CS, Henry RW, Huang S. TBP dynamics in living human cells, constitutive association of TBP with mitotic chromosomes. Mol Biol Cell 2002; 13:276-284.

Edited by Yun-Bo Shi 